\title{
Skeletal muscle weakness in older adults home-restricted due to COVID-19 pandemic: a role for full-body in-bed gym and functional electrical stimulation
}

\author{
Ugo Carraro ${ }^{1,2,3}$ (1) Andrea Marcante ${ }^{4} \cdot$ Barbara Ravara $^{1,2,3} \cdot$ Giovanna Albertin $^{2,5} \cdot$ Maria Chiara Maccarone $^{6}$. \\ Francesco Piccione ${ }^{7} \cdot$ Helmut Kern $^{8,9,10} \cdot$ Stefano Masiero ${ }^{2,7}$
}

Received: 3 February 2021 / Accepted: 9 May 2021 / Published online: 28 May 2021

(c) The Author(s), under exclusive licence to Springer Nature Switzerland AG 2021

\begin{abstract}
Persons suffering with systemic neuromuscular disorders or chronic organ failures, spend less time for daily physical activity, aggravating their mobility impairments. From 2020, patients at risk are also older adults, who, though negative for the SARS-Cov-2 infection, suffer with a fatigue syndrome due to home restriction/quarantine. Besides eventual psycological managements, it could be useful to offer to these patients a rehabilitation workouts easy to learn and to independently repeat at home (Full-Body In-Bed Gym). Inspired by the proven capability to recover skeletal muscle contractility and strength by home-based volitional exercises and functional electrical stimulation (FES), we suggest for this fatigue syndrome a 10-20 min long daily routine of easy and safe physical exercises that may recover from muscle weakness the main 400 skeletal muscles used for every-day activities. Leg muscles could be trained also by an adjunctive neuro-muscular electrical stimulation (NMES) in frail old persons. Many of the exercises could be performed in bed (Full-Body in-Bed Gym), thus hospitalized patients can learn this light training before leaving the hospital. Full-Body in-Bed Gym is, indeed, an extension of well-established cardiovascular-ventilation rehabilitation training performed by patients after heavy surgery. Blood pressure readings, monitored before and after daily routine of Full-Body in-Bed Gym, demonstrate a transient decrease in peripheral resistance due to increased blood flow to major body muscles. Continued regularly, Full-Body in-Bed Gym may help maintaining independence of frail people, including those suffering with the fatigue syndrome related to the restrictions/ quarantine imposed to the general population during the COVID-19 pandemic.
\end{abstract}

Keywords Skeletal muscle weakness · Home-based Full-Body in-Bed Gym · Borderline mobility impaired persons . Neuro-muscular electrical stimulation · COVID-19 fatigue syndrome

Ugo Carraro

ugo.carraro@unipd.it

1 Department of Biomedical Sciences, University of Padova, Via Ugo Bassi, 58/B, Padova, Italy

2 CIR-Myo-Interdepartmental Research Center of Myology, University of Padova, Padua, Italy

3 A\&C M-C Foundation for Translational Myology, Padua, Italy

4 UOC Recovery and Functional Rehabilitation, Lonigo Hospital, Azienda ULSS 8 Berica, Lonigo, Italy

5 Department of Neuroscience, Section of Human Anatomy, University of Padova, Padua, Italy
6 Physical Medicine and Rehabilitation School, University of Padova, Padua, Italy

7 Department of Neuroscience, Section of Rehabilitation, University of Padova, Padua, Italy

8 Ludwig Boltzmann Institute of Electrical Stimulation and Physical Rehabilitation, Vienna, Austria

9 Ludwig Boltzmann Institute of Rehabilitation Research, St Pölten, Austria

10 Institute of Physical Medicine and Rehabilitation, Prim. Dr. H Kern GmbH, Amstetten, Austria 
Roughly 400 human skeletal muscles are serious bone-movers, including little muscles of hands, feet, and face. The purpose of this report is to propose a comprehensive gym program to older adults, suffering skeletal muscle fatigue or with the outcomes from prolonged inactivity, to fight muscle atrophy and to maintain the best function and shape of body muscles [1]. Due to their advanced age and associated diseases, geriatric subjects spend little time on physical activity, in particular in the present situation of forced lockdown and quarantine imposed by the COVID-19 pandemic. Indeed, these subjects are, or risk to became, frail and at risk of falling, thus of losing and never recovering their previous physical and functional abilities.

The resulting disuse muscle atrophy further limits their independence, eventually forcing them to bed and hospitalization for long periods [2-4]. In addition to nutritional and pharmacological treatments, physical exercise at home is useful to counteract muscle atrophy in these persons [5-9].

Awaiting development of implantable devices for muscle stimulation, as effective as pacemakers for cardiac arrhythmias [10], implantable stimulators for ventilation supports $[11,12]$ or cochlear implants for hearing loss [13, 14], education of sedentary patients to perform home physical exercises could be an effective low-cost alternative during and after hospitalization [15-17]. Cardiovascular and ventilation physical rehabilitation protocols of surgical patients are well-established approaches, whose main goal is to reverse muscle weakness $[18,19]$. We extended those routines, to a daily short (10-20 min) sequence of easy-to-learn and safe volitional physical exercises to be performed in bed (FullBody in-Bed Gym) to improve muscles and, hence, mobility of impaired persons [15-17].

The risks are now even worsened by the conditions of lockdown and home restriction/quarantine imposed by the SARSCoV-2 pandemic. A fatigue syndrome related to COVID-19 disease is described in the general population, characterized by both the psychological response to the global problem of COVID-19 pandemic, and by muscle weakness that negatively influence the quality of life of persons for weeks or months after the resolution of an eventual SARS-CoV-2 infection [20-22].

In any case, muscle weakness can affect up to $10 \%$ of the population due to changes in people's ordinary lifestyle due to lockdown measures imposed to control the epidemics $[23,24]$. However, the most severe responses are expected later after resolution of an eventual SARS-CoV-2 infection. This post-COVID fatigue syndrome will affect the working capacity when economical recovery will be essential. Besides nutritional, pharmacological and psychotherapy support in the acute phases, it will be mandatory to control the mild cases by general prophylactic measures of healthy lifestyle, i.e., by normalization of sleep-wake schedule and moderate physical activity.

Here, our contribution is to convince practitioners [24-27], and the population at large that Full-Body in-Bed Gym is a valid option despite the minimal effort required [15-17] to counteract the effects of prolonged inactivity and persistent fatigue syndrome.

\section{Workout}

Figure 1 shows the exercises that could be a seasonal warmup also for active persons, i. e, those able to make at least 20 consecutive push-ups in $3 \mathrm{~min}$. On the other hand, sedentary people should start with five repetitions of each exercise, after advice from their practitioners to avoid exercise pain and eventual joint or muscle lesions. After two weeks, they should add groups of five additional repetitions, up to 20 , every additional couples weeks. If compliant, even geriatric patients will progressively increase their muscle strength, when reaching and maintaining 20 daily repetitions. It is advised to start performing the routine at very slow speed, but when the maximum number of each exercise is reached (20 repetitions), improving effects will be obtained by speeding up exercises and thus intensity. A video available at Youtube link: https://www.youtube.com/watch?v=N1RuG 3371-Y describes them dynamically [28].

\section{Theory}

Cautious Full-Body in-Bed Gym helps patients to recover earlier after hospitalization, reducing eventual arterial hypertension [29]. Indeed, after a routine that challenges personal fitness, i.e., induces forehead sweat, increase cardio-pulmonary rates, and maximal, but not minimal blood pressure, in a few minutes, the increased values return to those pre-excersise, together with minimal arterial pressure. Results of a week of trainings are exemplified in Fig. 2. Indeed, peripheral arterial resistance decreasesduring the series of challenging exercises when blood perfusion increases by functional hyperhemia of the main skeletal muscles [17]. Correlations between values of cardiac frequency and maximal arterial pressure are very high $\left(r^{2}<0.95\right)$ when the results of a week of Full-Body In-bed Gym are analyzed. In addition, within a few days of inpatient care, a Full-body In-Bed Gym program may increase muscle strength, fatigue resistance and independence in daily life activities [16]. Furthermore, Full-Body 


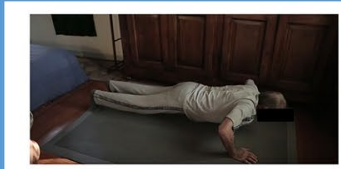

Exerc. 1. Push Up down

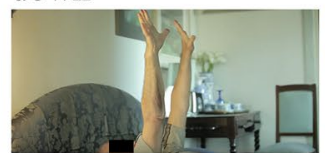

Exerc. 6 Hands up trunk

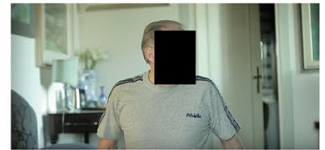

Exerc. 11 BendNeck Exerc. 12 Trunk up

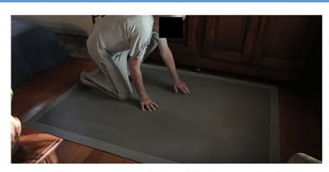

Exerc. 2. Stand Up

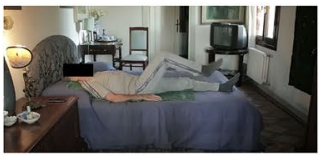

Exerc. 7 Leg cycling

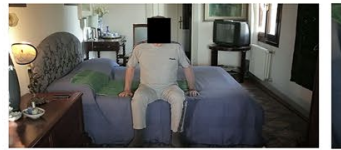

Exerc. 13 Leg up

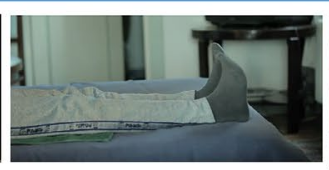

Exerc. 3 Flex Feet

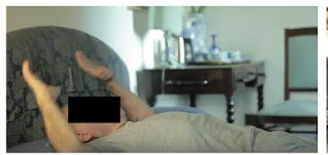

Exerc. 8 Deep breath
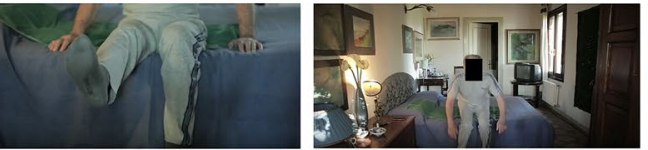

Exerc. 14. Stand Up

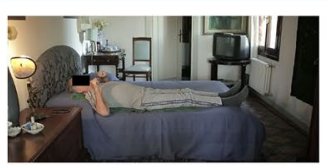

Exerc. 4 Extend Feet Exerc. 5 Hands
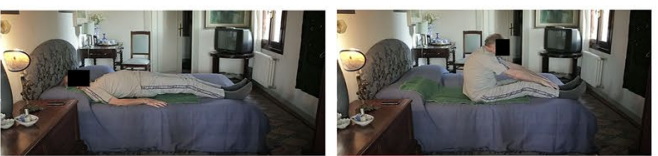

Exerc. 10 Bend

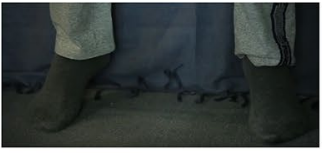

Exerc. 15. Up on
Fig. 1 The aim of the routine is to stimulate all main skeletal muscles, alternating exercises to mobilize arms and legs, spinal cord and neck, diaphragm and ventilation accessory muscles. The routine (as

in-Bed Gym routine mitigates the bad mood that is usually associated to mobility limitations [30]. If Full-Body in-Bed Gym is learned during hospitalisations, monitoring of the response to challenging trainings may include oxygen saturation and many more fitness variables. At home, using wearable devices (e.g. smartwatches, fitbands, smartphones, etc.) would guide during workout, providing heart rate monitoring and oxyhemoglobin saturation, reminder to perform exercises every day, and stopwatch for timing workout [31]. To speed up positive changes, persons are advised to the train twice a day to improve fatigue resistance and cardio-respiratory reserve [32,33].

During the initial learning period of Full-Body In-bed Gym, all older adults, if not hospitalized, are advised to be supervised by at least one trainer, if not a health professional to avoid harmful exercises, that are linked to personal fitness and, nothing to say, to comorbidities often present in the elderlies. Although different categories of older subjects with muscle weakness may benefit from a program of Full-Body In-bed Gym, specific pathways are provided for patients recovered from COVID-19 having persistent outcomes and for individuals who have not been infected but have been non-active for a long time (Fig. 3). number of repetitions and speed) is continuously increased in intensity up to fatigue threshold. A video: https://www.youtube.com/ watch? $\mathrm{v}=\mathrm{N} 1 \mathrm{RuG} 3371-\mathrm{Y}$ describes them dynamically [28]

Furthermore, all frail persons may benefit from the FullBody In-bed Gym program associated with electrical stimulation. If older subjects cannot, or are reluctant to perform volitional physical rehabilitation protocols, functional neuromuscular electrical stimulation (NMES) may mimic some of those exercises and be almost equally effective. NMES before or during the first weeks of workout is a rational approach for older adults to be submitted to Full-Body InBed Gym (Maria Chiara Maccarone, Padova, Italy, personal communication) or early rehabilitation after hip and knee surgical procedures (Helmut Kern, Vienna Austria, personal communication) [34-41].

Altogether, previous results demonstrated that physical exercise, either voluntary or induced by adjunctive NMES, improves functional performance of skeletal muscles, including those essential for ventilation, one of the main issues in post-COVID-19 old adults. Indeed, one of the most successful clinical applications of functional electrical stimulation (FES) is the respiratory support by pacing the diaphragm in quadriplegia and beyond [11, $12,42-47]$.

In conclusion, Full-Body in-Bed Gym could help older subjects with muscle weakness due to the pandemic-related 


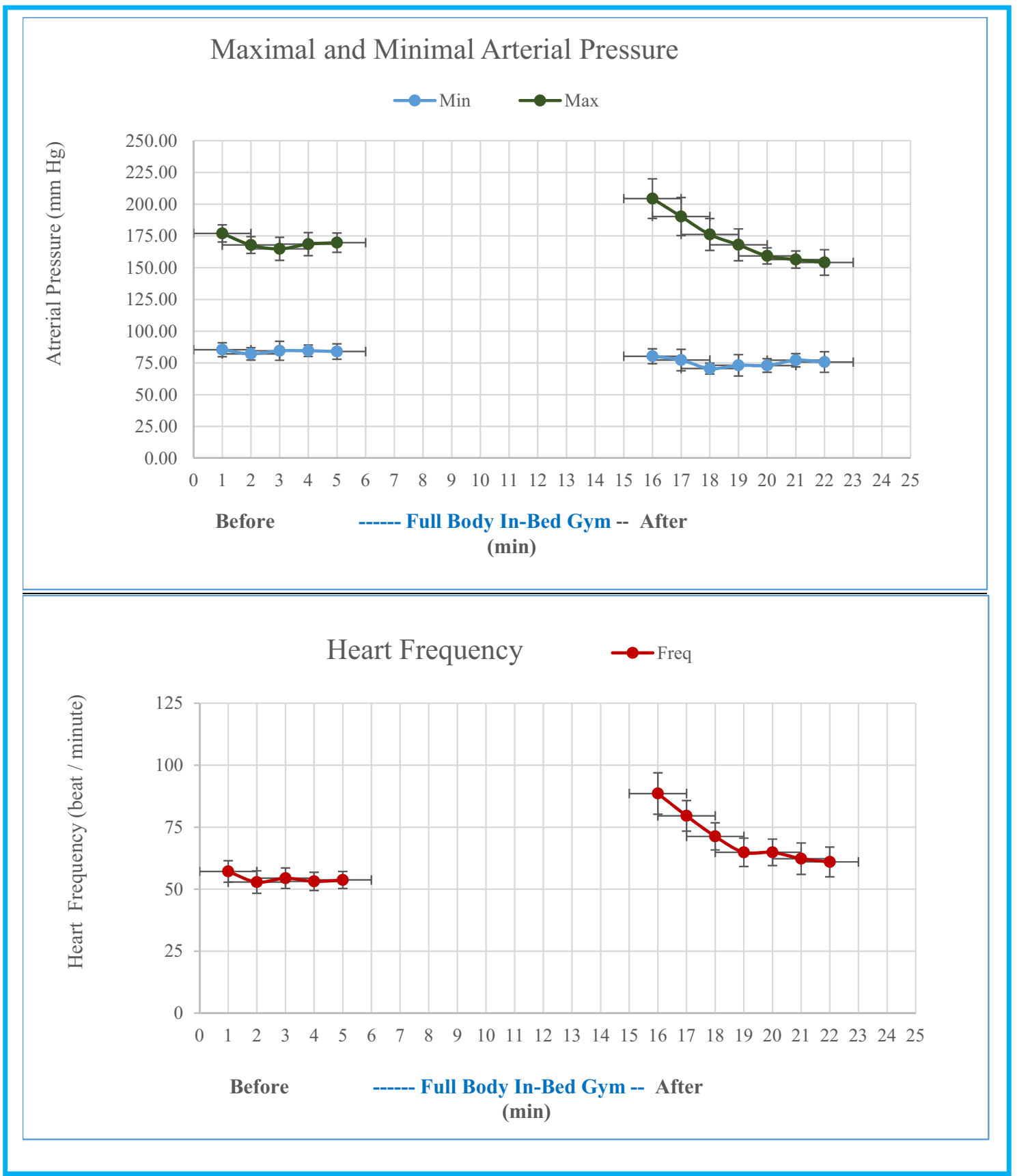

Fig. 2 Arterial pressure and cardiac frequency before and after 10 min of Full-Body In-Bed Gym (25 repetitions of each exercise) during seven consecutive days (November 8 to 16, 2016). Mean \pm SD 


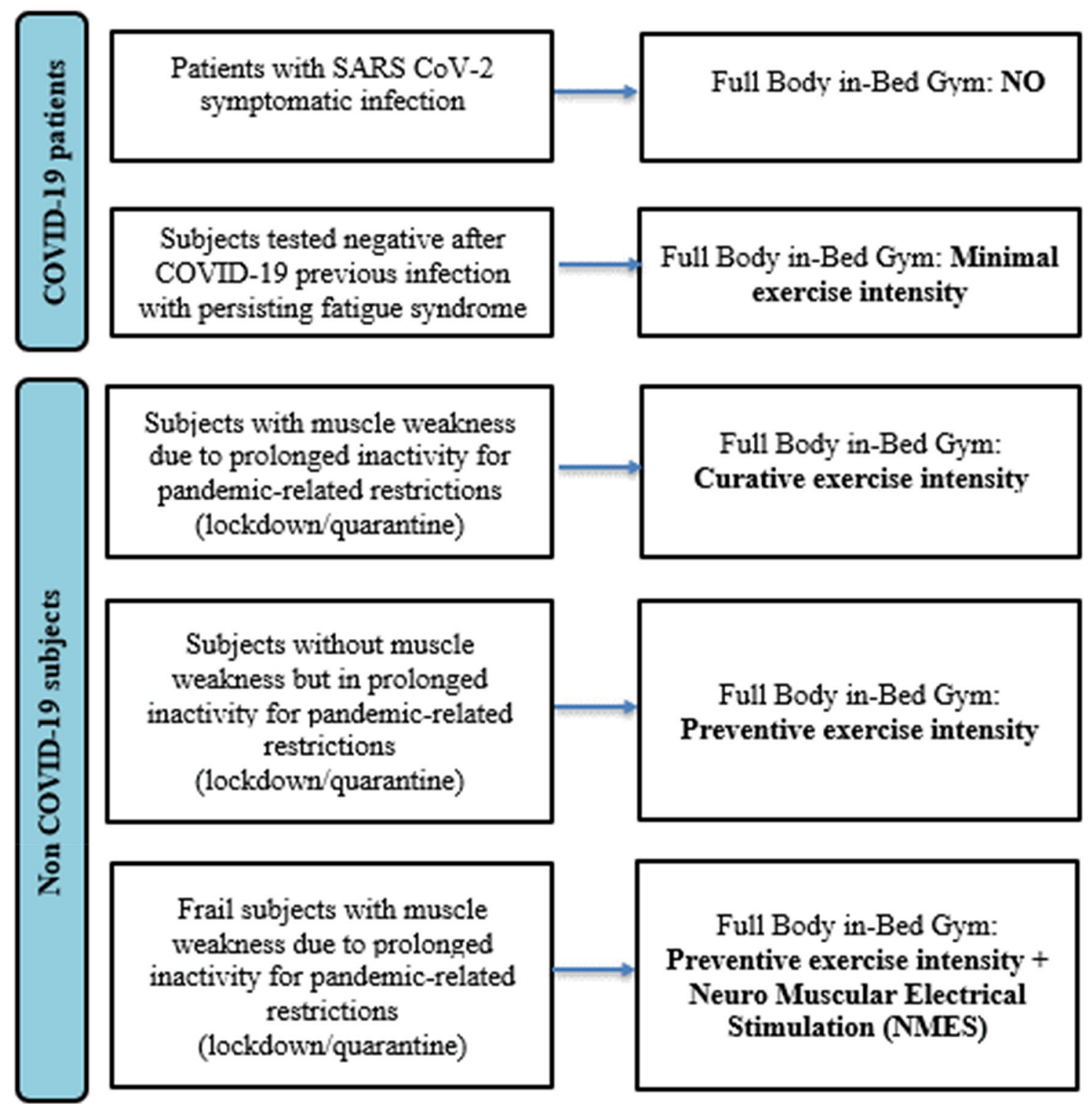

Fig. 3 Flow chart of older adults selection for Full-Body in-Bed Gym program and Neuro Muscular Electrical Stimulation in the COVID-19 scenario

inactivity, with the aim to prevent and recover them from skeletal muscle fatigue.

Funding A\&C M-C Foundation for Translational Myology, Padova, Italy sponsored publication of this typescript.

\section{Declartions}

Conflict of interest The authors state that there is no conflict of interest.

Human and animal rights Our study agreed with the Declaration of Helsinki for the study on human subjects and participants.
Informed consent Informed consent will be obtained from all individual participants who will be enrolled in future studies. No informed consent is required for this typescript.

\section{References}

1. Gava P, Kern H, Carraro U (2015) Age-associated power decline from running, jumping, and throwing male masters world records. Exp Aging Res 41:115-135. https://doi.org/10.1080/0361073X. 2015.1001648

2. Hopkins RO, Mitchell L, Thomsen GE et al (2016) Implementing a mobility program to minimize post-intensive care 
syndrome. AACN Adv Crit Care 27:187-203. https://doi.org/ 10.4037/aacnacc2016244

3. Camillo CA, Osadnik CR, Remoortel H et al (2016) Effect of "add-on" interventions on exercise training in individuals with COPD: a systematic review. ERJ Open Res 2:00078-02015. https://doi.org/10.1183/23120541.00078-2015

4. Czyrny JJ, Kaplan RE, Wilding GE et al (2010) Electrical foot stimulation: a potential new method of deep venous thrombosis prophylaxis. Vascular 18:20-27. https://doi.org/10.2310/6670. 2010.00001.ErratuminVascular2010Mar-Apr;18(2):121

5. Oshima K, Asai T, Fukumoto Y et al (2021) Development and persistence of fear of falling relate to a different mobility functions in community-dwelling older adults: one-year longitudinal predictive validity study. Aging Clin Exp Res. https://doi.org/10. 1007/s40520-020-01756-0

6. Rossi AP, Urbani S, Gattazzo S et al (2021) The Mini Sarcopenia Risk Assessment (MSRA) Questionnaire score as a predictor of skeletal muscle mass loss. Aging Clin Exp Res. https://doi.org/ 10.1007/s40520-020-01763-1

7. Sebastião E, Mirda D (2021) Group-based physical activity as a means to reduce social isolation and loneliness among older adults. Aging Clin Exp Res. https://doi.org/10.1007/ s40520-020-01722-w

8. Eckert T, Wronski P, Bongartz $\mathrm{M}$ et al (2021) Cost-effectiveness and cost-utility of a home-based exercise program in geriatric patients with cognitive impairment. Gerontology. https://doi. org/10.1159/000512748

9. Ebner SA, Meikis L, Morat M et al (2021) Effects of movementbased mind-body interventions on physical fitness in healthy older adults: a meta-analytical review. Gerontology. https://doi. org/10.1159/000512675

10. Saleem-Talib S, van Driel VJ, Chaldoupi SM et al (2019) Leadless pacing: going for the jugular. Pacing Clin Electrophysiol 42:395-399. https://doi.org/10.1111/pace.13607

11. Glenn WW, Hogan JF, Loke JS et al (1984) Ventilatory support by pacing of the conditioned diaphragm in quadriplegia. N Engl J Med 310:1150-1155. https://doi.org/10.1056/NEJM198405 033101804

12. Skalsky AJ, Lesser DJ, McDonald CM (2015) Evaluation of phrenic nerve and diaphragm function with peripheral nerve stimulation and M-mode ultrasonography in potential pediatric phrenic nerve or diaphragm pacing candidates. Phys Med Rehabil Clin N Am 26:133-143. https://doi.org/10.1016/j.pmr.2014.09. 010

13. Eskridge HR, Park LR, Brown KD (2021) The impact of unilateral, simultaneous, or sequential cochlear implantation on pediatric language outcomes. Cochlear Implants Int. https://doi.org/ 10.1080/14670100.2020.1871267

14. Gallo S, Trevisi P, Rigon C et al (2020) Auditory outcome after cochlear implantation in children with DFNB7/11 caused by pathogenic variants in TMC1 gene. Audiol Neurootol. https://doi.org/ $10.1159 / 000510156$

15. Carraro U, Karma Gava K, Baba A et al (2016) Fighting muscle weakness in advanced aging by takehome strategies: Safe antiaging full-body in-bed gym and functional electrical stimulation (FES) for mobility compromised elderly people. Biol Eng Med 1:1-4. https://doi.org/10.15761/BEM.1000106

16. Carraro U, Gava K, Musumeci A et al (2018) Safe antiaging Full-Body In-Bed Gym and FES for lazy persons: home in-bed exercises for fighting muscle weakness in advanced age. In: Masiero S, Carraro U (eds) Rehabilitation medicine for elderly patients, pp 43-52. ISBN 978-3-319-57405-9 ISBN 978-3-31957406-6. https://doi.org/10.1007/978-3-319-57406-6

17. Carraro U, Gava K, Baba A et al (2018) To contrast and reverse skeletal muscle atrophy by full-body in-bed gym, a mandatory lifestyle for older olds and borderline mobility-impaired persons. Adv Exp Med Biol 1088:549-560. https://doi.org/10. 1007/978-981-13-1435-3_25

18. Ades PA, Keteyian SJ, Wright JS et al (2017) Increasing cardiac rehabilitation participation from 20\% to 70\%: a road map from the million hearts cardiac rehabilitation collaborative. Mayo Clin Proc 92:234-242. https://doi.org/10.1016/j.mayocp.2016. 10.014

19. Vorona S, Sabatini U, Al-Maqbali S et al (2018) Inspiratory muscle rehabilitation in critically ill adults: a systematic review and meta-analysis. Ann Am Thorac Soc 15:735-744. https://doi. org/10.1513/AnnalsATS.201712-961OC

20. Soloveva NV, Makarova EV, Kichuk IV (2020) Coronavirus syndrome: COVID-19 psychotrauma. Eur J Transl Myol 30:9302. https://doi.org/10.4081/ejtm.9302

21. Khosravi M (2020) COVID-19 quarantine: two-way interaction between physical activity and mental health. Eur J Transl Myol 30:9509. https://doi.org/10.4081/ejtm.2020.9509

22. Angelini C, Siciliano G (2020) Neuromuscular diseases and Covid-19: advices from scientific societies and early observations in Italy. Eur J Transl Myol 30:9032. https://doi.org/10. 4081/ejtm.2019.9032

23. Moro T, Paoli A (2020) When COVID-19 affects muscle: effects of quarantine in older adults. Eur J Transl Myol 30:069. https:// doi.org/10.4081/ejtm.2019.9069.eCollection2020Jul13.PMID: 32782767FreePMCarticle

24. O'Hara J (2020) Rehabilitation after COVID-19. Mayo Clinic News Network. Available at the link: https://newsnetwork. mayoclinic.org/discussion/rehabilitation-after-covid-19/

25. Demeco N, Marotta M, Barletta I et al (2020) Rehabilitation of patients post-COVID-19 infection: a literature review. J Int Med Res 48:300060520948382. https://doi.org/10.1177/0300060520 948382

26. Wang TJ, Chau B, Lui M et al (2020) Physical medicine and rehabilitation and pulmonary rehabilitation for COVID-19. Am J Phys Med Rehabil 99:769-774. https://doi.org/10.1097/PHM. 0000000000001505

27. Smith JM, Lee AC, Zeleznik H et al (2020) Home and community-based physical therapist management of adults with postintensive care syndrome. Phys Ther 100:1062-1073. https:// doi.org/10.1093/ptj/pzaa059.PMID:32280993;PMCID:PMC71 88154

28. Available at Youtube link: https://www.youtube.com/watch?v= N1RuG3371-Y

29. Börjesson M, Onerup A, Lundqvist S et al (2016) Physical activity and exercise lower blood pressure in individuals with hypertension: narrative review of 27 RCTs. Br J Sports Med 50:356-361. https://doi.org/10.1136/bjsports-2015-095786

30. Carneiro LS, Fonseca AM, Serrão P et al (2016) Impact of physical exercise on catechol-O-methyltransferase activity in depressive patients: a preliminary communication. Affect Disord 193:117-122. https://doi.org/10.1016/j.jad.2015.12.035

31. Graña Possamai C, Ravaud P, Ghosn L et al (2020) Use of wearable biometric monitoring devices to measure outcomes in randomized clinical trials: a methodological systematic review. BMC Med 18:310. https://doi.org/10.1186/s12916-020-01773-w

32. Masiero S, Maccarone MC, Agostini F (2020) Health resort medicine can be a suitable setting to recover disabilities in patients tested negative for COVID-19 discharged from hospital? A challenge for the future. Int J Biometeorol 64:1807-1809. https://doi.org/10.1007/s00484-020-01947-4

33. Masiero S, Zampieri D, Del Felice A (2020) The place of early rehabilitation in intensive care unit for COVID-19. Am J Phys Med Rehabil 99:677-678. https://doi.org/10.1097/PHM.00000 00000001478

34. Kern H, Carraro U, Adami N et al (2010) Home-based functional electrical stimulation rescues permanently denervated 
muscles in paraplegic patients with complete lower motor neuron lesion. Neurorehabil Neural Repair 24:709-721. https://doi. org/10.1177/1545968310366129

35. Carraro U, Kern H (2016) Severely atrophic human muscle fibers with nuclear misplacement survive many years of permanent denervation. Eur J Trans1 Myol 26:5894. https://doi.org/ 10.4081/ejtm.2016.5894

36. Carraro U, Kern H, Gava P et al (2015) Biology of muscle atrophy and of its recovery by FES in aging and mobility impairments: roots and by-products. Eur J Transl Myol 25:221-230. https://doi.org/10.4081/ejtm.2015.5272

37. Kern H, Barberi L, Löfler S et al (2014) Electrical stimulation counteracts muscle decline in seniors. Front Aging Neurosci 24:189. https://doi.org/10.3389/fnagi.2014.00189

38. Carraro U, Kern H, Gava P et al (2017) Recovery from muscle weakness by exercise and FES: lessons from Masters, active or sedentary seniors and SCI patients. Aging Clin Exp Res 29:579-590. https://doi.org/10.1007/s40520-016-0619-1

39. Zampieri S, Mosole S, Löfler S et al (2015) Physical exercise in aging: nine weeks of leg press or electrical stimulation training in 70 years old sedentary elderly people. Eur J Transl Myol 25:237-242. https://doi.org/10.4081/ejtm.2015.5374

40. Kern H, Carraro U (2020) Home-based functional electrical stimulation of human permanent denervated muscles: a narrative review on diagnostics, managements, results and byproducts revisited 2020. Diagnostics (Basel) 10:529. https://doi.org/ 10.3390/diagnostics 10080529

41. Recenti M, Ricciardi C, Edmunds K et al (2020) Machine learning predictive system based upon radiodensitometric distributions from mid-thigh CT images. Eur J Transl Myol 30:8892. https://doi.org/10.4081/ejtm.2019.8892
42. Albertin G, Ravara B, Kern H et al (2019) Two-years of home based functional electrical stimulation recovers epidermis from atrophy and flattening after years of complete Conus-Cauda Syndrome. Medicine (Baltimore) 98:e18509. https://doi.org/ 10.1097/MD.0000000000018509

43. Zampieri S, Pietrangelo L, Loefler S et al (2015) Lifelong physical exercise delays age-associated skeletal muscle decline. J Gerontol A Biol Sci Med Sci 70:163-173. https://doi.org/10. 1093/gerona/glu006

44. Glenn WW, Phelps ML (1985) Diaphragm pacing by electrical stimulation of the phrenic nerve. Neurosurgery 17:974-984. https://doi.org/10.1227/00006123-198512000-00021

45. Berger D, Bloechlinger S, von Haehling S et al (2016) Dysfunction of respiratory muscles in critically ill patients on the intensive care unit. J Cachexia Sarcopenia Muscle 7:403-412. https://doi.org/10.1002/jcsm.12108

46. Peñuelas O, Keough E, López-Rodríguez L et al (2019) Ventilator-induced diaphragm dysfunction: translational mechanisms lead to therapeutical alternatives in the critically ill. Intensive Care Med Exp 7:48. https://doi.org/10.1186/s40635-019-0259-9

47. Marrero HDJG, Stålberg EV, Cooray G et al (2020) Neurogenic vs. myogenic origin of acquired muscle paralysis in intensive care unit (ICU) patients: evaluation of different diagnostic methods. Diagnostics (Basel). 10:966. https://doi.org/10.3390/diagn ostics 10110966

Publisher's Note Springer Nature remains neutral with regard to jurisdictional claims in published maps and institutional affiliations. 